\title{
Opportunistic Message Routing using Multi-layer Social Networks
}

\author{
Annalisa Socievole \\ DIMES Department \\ University of Calabria \\ Rende (CS), Italy \\ socievolea@deis.unical.it
}

\author{
Eiko Yoneki \\ Computer Laboratory \\ University of Cambridge \\ Cambridge, UK \\ eiko.yoneki@cl.cam.ac.uk \\ Jon Crowcroft \\ Computer Laboratory \\ University of Cambridge \\ Cambridge, UK \\ jon.crowcroft@cl.cam.ac.uk
}

\author{
Floriano De Rango \\ DIMES Department \\ University of Calabria \\ Rende (CS), Italy \\ derango@deis.unical.it
}

\section{Keywords}

Delay tolerant network; opportunistic network; online social network; multi-layer network

\section{INTRODUCTION}

The diffusion of mobile devices carried by users, such as smartphones, has led to a growing interest in novel infrastructureless network architectures exploiting peer-to-peer opportunistic connectivity. In a world where people are becoming increasingly reliant on mobile communication in several aspects of their life, being unable to communicate can negatively affect both business and personal relationships. An alternative system is therefore necessary where there is no suitable network architecture.

Delay Tolerant Networks (DTNs) [8, 3] were developed to allow communication in scenarios where fixed infrastructure is not available and existing IP and GSM/UMTS network protocols are unsuitable. In such scenarios, where nodes often create sparse network topologies and the contacts between them are intermittent, DTNs use a storecarry-forward strategy to allow communication when a path through the network is not reliable, due to frequent disconnections. A node receiving a packet from one of its contacts can buffer the message, carry it while moving, and forward it to the encountered nodes which are at least as useful as itself in terms of delivery. A network that routes packets using the store-carry-forward approach is also called opportunistic network [17], because nodes forward messages when the opportunity arises: during an encounter contact. The networks formed by mobile handheld devices exploiting human mobility for opportunistic forwarding are also known as Pocket Switched Networks (PSN) [10].

In the research field on opportunistic network routing, many works are related to the best way to select relay nodes considering social interactions to optimize message delivery. Commonly, the social information is extracted from encounters between mobile devices detected through wireless interfaces such as Bluetooth, ZigBee and Wi-Fi $[11,14,5,1$, $23,6]$. However, other social interaction techniques that are not based on physical meetings provide us with insights into user behavior such as email, chats and online social networks services (e.g. Facebook, Twitter, MySpace, and LinkedIn) 
$[9,21]$. While opportunistic contact information changes constantly and it takes time to identify an optimal relay node which has to forward a message towards the destination, online social network ties represent explicitly declared and stable relationships, and can be used to augment available partial contact information. For this reason, we believe that by designing routing metrics that combine social network information discovered through encounters with social information extracted from other social network layers, the chances of the message reaching its destination can be maximized. The more additional social network information is available, the more likely the optimal forwarding decision can be made, since we have a more accurate and complete view of the social behavior of each node.

The aim of this paper is to demonstrate that the use of social information extracted from multiple social networks improves message delivery in opportunistic networks. Although some forwarding schemes $[15,19,16,2,7,4,20]$ using both online social network and detected social network information have been proposed to increase the delivery speed and the likelihood of a message to reach its destination, our approach to exploit more than two social network layers has not been addressed before. We propose a Multi-Layer Social network based Opportunistic Routing (ML-SOR) protocol, in which a node forwards packets using a routing metric that combines three measures: node centrality, tie strength and a tie predictor. Each one of these measures is computed on a different social network layer. We evaluate our algorithm using two mobility traces and three corresponding social network layers: (1) contact network, (2) Facebook network and (3) Interest network. In order to test the effectiveness of ML-SOR, we compare our scheme to Epidemic routing[22], PRoPHET [13], Bubble Rap [11], and H-Bubble Rap, a hybrid version of Bubble Rap computing centrality on a multi-layer social network. We define this hybrid version of Bubble Rap in order to obtain another social-based protocol extracting social information from a multi-layer social network. The results show that ML-SOR achieves message delivery ratio similar to Epidemic Routing with significantly lower overhead cost.

The remainder of the paper is organized as follows. Section 2 provides a review of existing works in this area. Section 3 formally defines a multi-layer social network. Section 4 presents ML-SOR, our multi-layer social network based routing proposal. Section 5 details the datasets, the algorithms in comparison and the performance metrics used to test ML-SOR. Section 6 presents the results of performance evaluation. Finally, Section 7 discusses the results and section 8 concludes the paper.

\section{RELATED WORK}

In opportunistic networks, the design of efficient forwarding schemes is one of the main challenges. Social-based routing protocols make use of human behavior characteristics to optimize data delivery. Most of these protocols use community structure and/or centrality as routing metrics, and compute them on social graph detected through real-world contacts between mobile devices. We refer to this proximity graph as detected social network (DSN). Only few works drive routing decision also using social information extracted from virtual or self-declared contacts. We call the social network based on these kind of contacts online social network (OSN).
In the following subsections we describe the main socialbased forwarding schemes for opportunistic networks, by dividing them into two categories; using only DSN or using both DSN and OSN for extracting social routing metrics.

\subsection{Opportunistic forwarding using only DSN}

Bubble Rap [11] is a social-based protocol using two centrality values that are associated to each node based on the node global popularity in the whole network and local popularity within its community or communities. The forwarding scheme uses these centrality values so that a message is transferred to nodes with higher global centrality values until the carrier node meets a node with the same community label as the destination node. A message is forwarded to nodes with higher local rankings until successful delivery. Habit [14] realizes data dissemination in a selection-based manner by exploiting node physical proximity and user social ties. A regularity graph is used to keep trace of when and how often two nodes come into contact, and an interest graph is used to build dissemination paths based on nodes interested in the data they are willing to route. In SimBetTS [5] routing protocol, a node forwards a message to an encountered node according to three social metrics: betweenness (the number of shortest paths on which a node lies), similarity (the number of ties that two nodes share), and tie-strength (the recency, duration and number of contacts between two nodes). When two nodes meet, they exchange a list of encountered nodes, used to locally calculate the betweenness utility, the tie strength utility and the similarity utility. Each node then examines the messages it is carrying and computes the overall utility value of each message destination. Messages are then forwarded to the node holding the highest overall utility for the message destination node. In [1] and [23], the popularity of a node in DSN is exploited for message forwarding. In the former, popular nodes (called hubs) are those connected with most of the other nodes in DSN and are characterized by analyzing the history of encounters. In the latter, a destination-unaware message-forwarding strategy that takes into consideration both the popularity of a node in DSN and the contact durations is proposed.

\subsection{Opportunistic forwarding using both DSN and OSN}

In [15], a Bluetooth-based mobile social network application deployed among a group of participants during a computer communication conference is analyzed and it is shown that the structure of the social graph constructed on selfdeclared friends helps to build forwarding paths in the contact graph, allowing two nodes to communicate over time using opportunistic contacts and intermediate nodes. In $\mathrm{Mo}$ biClique [19], a novel mobile social networking middleware that uses the Facebook network to bootstrap the opportunistic network is proposed. This middleware leverages DSN and OSN so that users can move between them in a way that enhances both. During an opportunistic encounter, if the two user profiles are friends or share some interests, the users are alerted and can choose to exchange messages. In [2], an opportunistic routing protocol, called Social Role Routing (SRR), that uses OSN information to bootstrap the opportunistic network is presented. SRR categorizes nodes into roles by applying the social network analysis technique of regular equivalence that partitions nodes into classes, where 
all nodes in a class are connected to the same classes of nodes. Before the network starts up, each node stores a copy of a role connectivity graph, which has been precomputed using the OSN of the participating nodes, allowing them to compute the geodesic distance between roles. Messages are then forwarded only to intermediate nodes that are in the same role, or in a role adjacent to the destination's role. PeopleRank [16] makes use of OSN in a different way. In the OSN graph (called social graph), a social relationship between two nodes is defined either if the nodes are declared friends, or if they share interests. This information is used by the routing scheme to compute node rankings. PeopleRank gives higher weight to nodes if they are socially connected to other important nodes of the network. When two neighbor nodes in the OSN graph meet, they exchange two pieces of information: their current PeopleRank values and the number of OSN neighbors they have. Messages are then forwarded towards nodes with a higher PeopleRank value. In SPRINT [4], OSN information is combined with contact history and predictions of future encounters in order to optimize opportunistic routing. All these aspects are used by each SPRINT node to compute utility values for its messages and perform social-based routing accordingly. Message utility is computed considering the freshness and the number of hops of the message, the delivery probability and the popularity of the carrier node, the future meeting, the social connection and the time spent between the carrier and the destination.

\section{MULTI-LAYER SOCIAL NETWORK MODEL}

DSN and OSN described above represent two different social contexts. The former deals with physical encounters while the latter is related to virtual contact between nodes. If we extend the number of social contexts and consider several social networks for a particular set of users, we obtain a multi-layer structure, representing the connections of a single user to other users on several autonomous layers. Two users might be connected by many layers at the same time e.g. two users may be connected through Bluetooth network, Facebook, LinkedIn and Twitter networks - while other users may be connected on just one layer - e.g. like co-workers connected only through LinkedIn or friends only through Facebook. The result is a complex system where there are several social network layers and where users exploit different kind of relationships. Our definition of a multi-layer social network model is based on weighted graphs, where edge weights can be used to represent the strength of the relationship.

Definition 1. A social network layer $L$ is a weighted graph $G(V, E)$ with vertex set $V$ corresponding to users on the social network and and edge set $E \subseteq V \times V$ corresponding to social links between users.

Definition 2. A multi-layer social network is a tuple $M L S N=\left(L_{1}, L_{2}, \ldots, L_{n}\right)$ where $L_{i}=G_{i}\left(V, E_{i}\right), i \in 1, \ldots, n$ are social network layers.

An example of multi-layer social network is shown in Fig. 1. In the following sections, we will demonstrate the effectiveness of using such network for message forwarding in opportunistic networks.

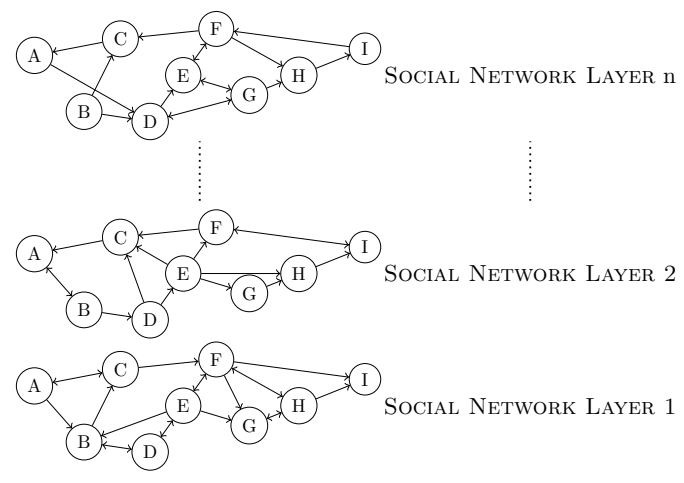

Figure 1: A multi-layer social network.

\section{ML-SOR: OPPORTUNISTIC ROUTING WITH MULTIPLE SOCIAL NETWORK LAYERS}

Because of the unpredictability and the temporal dynamics of contacts among users, it takes time to infer the corresponding social behavior. Thus, routing protocols using social information emerging from encounters may produce sub-optimal forwarding paths. In this section, we present ML-SOR, a multi-layer social network based opportunistic routing scheme exploiting the knowledge of real-word interactions, online connections and interests between individuals.

\subsection{ML-SOR social metric}

ML-SOR is based on a social metric which exploits social information extracted from different network layers. This metric is calculated using a combination of three measures:

- centrality on DSN layer

- tie strength on OSN layer(s)

- link predictor on Interest network layer

Centrality in graph theory and network analysis quantifies the structural importance of a vertex within the graph. Typically, a central node has a stronger capability of connecting other network members. We therefore consider node centrality as the most important factor to decide whether a node is a good next hop. ML-SOR computes centrality at the DSN layer, where the corresponding social graph is leveraged through encounters between mobile devices. ML-SOR social metric computes node centrality for a node $i, C_{C D e g r e e}(i)$, using a long-term cumulative estimate of degree centrality. Degree centrality basically counts how many connections a node has. We choose this measure since it can be easily computed locally considering only a node's ego network, while other centrality measures (e.g. betweenness, closeness or eigenvector centrality) require global knowledge of the network (for more details on these measures, see, e.g., [21]). More specifically, ML-SOR sets a time slot so that nodes can compute the number of unique nodes seen throughout this interval and then average this measure with a set of previous measures. Degree centrality for a node $i$ during a 
time slot $t$ is computed as follows:

$$
C_{\text {Degree }}(i, t)=\sum_{j=1}^{N} e(i, j, t)
$$

where

$$
e(i, j, t)=\left\{\begin{array}{cc}
1 & \text { if } i \text { encounters } j \text { during time slot } t \\
0 & \text { otherwise }
\end{array}\right.
$$

represents an edge between node $i$ and node $j$ on the DSN graph corresponding to the time slot considered ${ }^{1}$, and $N$ is the number of nodes in $i$ 's range. $C_{C D e g r e e}(i)$ is then calculated as the node's average degree over a set of $T$ time slots including the most recent time slot and all the previous ones:

$$
C_{C \text { Degree }}(i)=\frac{1}{T} \sum_{t=0}^{T} C_{\text {Degree }}(i, T-t)
$$

In that way, ML-SOR provides a fully decentralized approximation for a node's degree centrality.

Considering that centrality is measured using the contact history and does not account for future links availability, we include a tie strength indicator, which identifies links that have a higher probability to be activated, into ML-SOR social metric. Social ties on online social networking websites, such as Facebook, Twitter ${ }^{2}$ or LinkedIn, are more stable and hence stronger than contact network ties. Consequently, they can be considered a good measure of whether a tie will be activated. ML-SOR calculates tie strength between a node $i$ and a node $j$ at online social network layer $l$ as:

$$
T S(i, j, l)=\left\{\begin{array}{cc}
1 & \text { if } i \text { and } j \text { are connected at layer } l \\
0 & \text { otherwise }
\end{array}\right.
$$

The total tie strength between two nodes is the sum of the indicators measured at each online social network layer:

$$
T S_{T O T}(i, j)=\sum_{l=1}^{L} T S(i, j, l)
$$

where $L$ is the total number of online social networking websites considered.

ML-SOR social metric takes into account a third measure useful to predict future collaborations between two nodes. A link predictor is computed on an interest network layer, where a link between two nodes exists if they have at least one interest in common. Examining common neighbors of a pair of nodes at interest network layer, we can predict a future interaction between them. If two nodes have one or more common neighbors, the probability of future collaboration increases. ML-SOR computes the link predictor $L P(i, j)$ of a possible future collaboration between node $i$ and node $j$ as a common neighbor measure based on Jaccard index:

$$
L P(i, j)=\frac{|N(i) \cap N(j)|}{|N(i) \cup N(j)|}
$$

where $N(i)$ and $N(j)$ are the number of neighbors of node $i$ and the number of neighbors of node $j$, respectively.

\footnotetext{
${ }^{1}$ Considering that the DSN graph is a temporal graph, we form a static graph for each time slot by amalgamating all contacts detected in that time interval.

${ }^{2}$ Here we consider a tie between a user $\mathrm{A}$ and a user $\mathrm{B}$, if $\mathrm{A}$ follows B and vice versa
}

For each measure, ML-SOR determines the utility score of node $i$ for delivering a message to node $d$ compared to node $j$ as follows:

$$
\begin{gathered}
C S(i, j)=\frac{C_{C \text { Degree }}(i)}{C_{\text {Degree }}(i)+C_{C \text { Degree }}(j)} \\
T S S(i, j, d)=\frac{T S_{T O T}(i, d)}{T S_{T O T}(i, d)+T S_{T O T}(j, d)} \\
\operatorname{LPS}(i, j, d)=\frac{L P(i, d)}{L P(i, d)+L P(j, d)}
\end{gathered}
$$

The ML-SOR social metric is given by the sum of the contributing score values as follows:

$$
M L S(i, j, d)=C S(i, j)[1+T S S(i, j, d)+L P S(i, j, d)]
$$

As can be observed, MLS captures the relay significance of a node when compared to an encountered node across all social network layers, in terms of centrality, tie strength and link predictor. In particular, the social metric is given by the sum of centrality utility score with tie strength and tie predictor utility scores weighted with centrality utility score. In that way, we consider centrality as the predominant factor in message forwarding.

\subsection{ML-SOR forwarding strategy}

The forwarding process in ML-SOR is based on the comparison of the $M L S$ social metric. When two nodes meet, they exchange their centrality values, one or more lists of online social contacts (one list for each online social networking website) and a list of nodes with common interests. Each node then examines the messages it is carrying and computes the $M L S$ social metric of each message destination. Messages are then forwarded to the nodes with higher $M L S$ value for the message destination node. The proposed forwarding scheme is given by Algorithm 1.

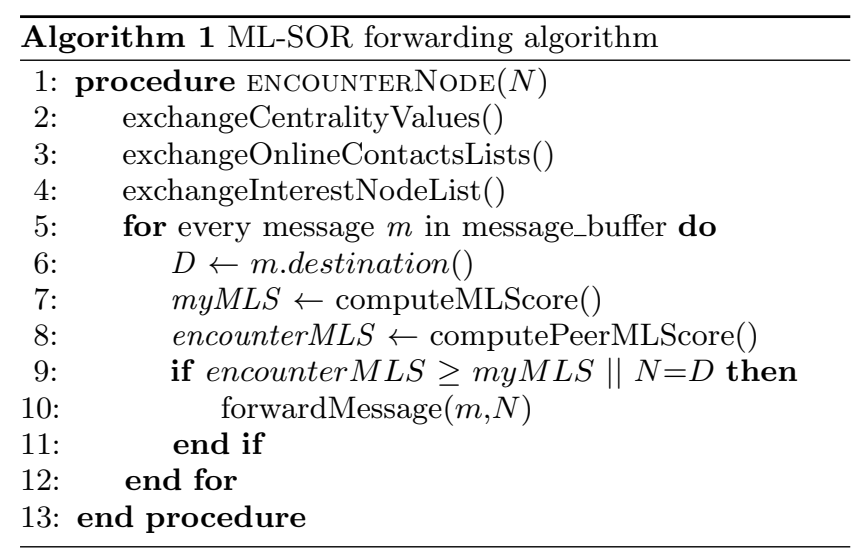

\section{EVALUATION}

We evaluate the performance of ML-SOR on real-world traces and compare it to other benchmark opportunistic routing algorithms. Our simulations are carried out on the Opportunistic Network Environment (ONE) simulator [12], a specific simulation tool for opportunistic networks. In this section, we first describe the experimental datasets, the algorithms in comparison and the performance metrics used in the simulations, and then present the results of performance evaluation. 


\subsection{Experimental datasets}

To test our proposal on realistic settings, we use two experimental datasets of human mobility also containing the Facebook friendlists of the participants and their interests: Lapland [24] and Sigcomm [18]. Table 1 summarizes their main characteristics. In these experiments, participants carry mobile devices that periodically discover others in Bluetooth proximity range and log contacts. For both datasets we consider a multi-layer social network formed by the following layers: (1) Bluetooth contact network, (2) Facebook network and (3) Interest network.

Fig. 2 shows the contact duration and the total number of contacts distributions. As can be seen, they follow both an approximate power law in each dataset. By looking at the complementary CDF of contact durations, $52 \%$ of Lapland contact durations last more than one hour, while only $4 \%$ last more than 3 hours. In Sigcomm, contact durations are shorter. Only $5 \%$ of contact durations last more than 1 hour. By looking at the number of contacts in Lapland, it is possible to observe that $50 \%$ of the number of contacts is greater than 26, and $15 \%$ is greater than 50. In Sigcomm, on the contrary, the number of contact opportunities between node pairs is significantly lower. Only $10 \%$ of the number of contacts is greater than 10 .

\subsection{Routing algorithms}

Considering that there are many forwarding methods in the literature for opportunistic networks, we choose those that we consider most relevant for the comparison with MLSOR. We test three popular routing methods for doing the simulations, Epidemic routing[22], PRoPHET [13] and Bubble Rap, together with another scheme, H-Bubble Rap, that is a hybrid version of Bubble Rap computing centrality on a multi-layer social network. In the following lines, a brief explanation motivates the reason for which we consider them relevant for the comparison with ML-SOR:

- Epidemic routing: this method has been chosen because of its flooding-based strategy. When two node encounter, they exchange all of their messages. In such way, messages spread like viruses by pairwise contacts between two nodes. This protocol can be considered a reference for other routing methods, since it determines an upper bound for message delivery.

- PRoPHET: it is a probabilistic routing method that calculates a metric, named delivery predictability, based on contact histories. A node that is carrying a message, relays it only to a node with higher deliver predictability. This is a well known protocol in oppor-

Table 1: Characteristics of the datasets.

\begin{tabular}{|l|l|l|}
\hline & Lapland & Sigcomm \\
\hline DSN type & Bluetooth & Bluetooth \\
\hline Radio range & $10 \mathrm{~m}$ & {$[10-20] \mathrm{m}$} \\
\hline \# of devices & 17 & 76 \\
\hline Device type & phone & phone \\
\hline Trace duration & $399812 \mathrm{~s}$ & $320593 \mathrm{~s}$ \\
\hline Granularity & {$[120-600] \mathrm{s}$} & $120 \pm 10 \mathrm{~s}$ \\
\hline OSN type & Facebook & Facebook \\
\hline OSN \# of edges & 47 & 66 \\
\hline Interests type & scientific & Facebook \\
\hline Interest network \# of edges & 157 & 1536 \\
\hline
\end{tabular}
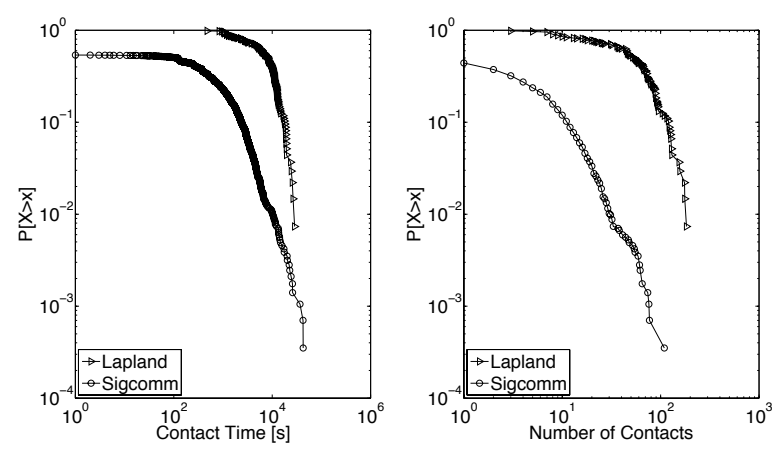

Figure 2: Complementary CDFs of contact durations and the number of contacts.

Table 2: Values for the simulation parameters.

\begin{tabular}{|l|l|l|}
\hline & Parameter & Value \\
\hline Network & Buffer size & $2000 \mathrm{MB}$ \\
\hline & Message size & $1 \mathrm{kB}$ \\
\hline & Inter-message creation interval & $1800 \mathrm{~s}$ \\
\hline PRoPHET & $P_{\text {init }}$ & 0.75 \\
\hline & $\beta$ & 0.25 \\
\hline & $\gamma$ & 0.98 \\
\hline Bubble Rap & C-Window duration & 6 hours \\
\hline & C-Window \# of windows & 5 \\
\hline ML-SOR & K (K-Clique) & 5 \\
\hline & Time slot & 6 hours \\
\hline & T & 5 \\
\hline
\end{tabular}

tunistic networks and it is usually used, as Epidemic, in comparisons.

- Bubble Rap: as previously described, this is a socialbased routing method which uses centrality and community as routing metrics. We choose this method to compare ML-SOR to another social-based forwarding strategy where centrality is identified as the metric with a dominant impact on routing.

- H-Bubble Rap: this is a hybrid version of Bubble Rap that we implemented in order to obtain another socialbased protocol extracting social information from a multi-layer social network. Local centrality and global centrality metrics are replaced with $M L S$ metric computed with local $C_{C \text { Degree }}$ and $M L S$ metric computed with global $C_{C D e g r e e}$, respectively.

\subsection{Performance metrics}

We analyze the following commonly used metrics to evaluate these algorithms: delivery ratio (the ratio of the number of delivered packets to the number of all packets), overhead cost (the number of packets transmitted across the air divided by the number of unique packets created), and average latency (the average time it takes a packet to be delivered). We evaluate all these metrics as a function of TTL: the maximum time a message can stay in the system after its creation. This is a fundamental parameter for studying the ability of a routing protocol to find the necessary number of relays within a certain time. In Table 2, we specify the common simulation parameters used for all the simulations. 


\section{RESULTS}

In this section, we present the results obtained after performing the simulations. We first evaluate the utility of the three metrics computed at different social network layers in terms of delivery ratio. Then, we illustrate ML-SOR routing behavior compared to the other routing algorithms in Lapland and Sigcomm datasets, respectively, having different characteristics in number of nodes, duration, mobility patterns and social dynamics.

\subsection{Evaluation of social metrics at different social network layers}

We start evaluating the routing performance of each MLSOR utility score and the benefit of combining the three utility scores in order to improve message delivery. Fig 3 shows the average delivery ratio for Lapland and Sigcomm datasets after simulating several scenarios with different TTL values. As can be observed, when evaluating centrality, tie strength and tie predictor utility scores, routing based on DSN centrality has the highest delivery performance both in Lapland and in Sigcomm. This result confirms that centrality is the most important factor in message forwarding. By combining centrality with the other two metrics, we can note that the overall delivery ratio increases, especially in Sigcomm dataset where ML-SOR achieves $88 \%$ of delivery ratio while routing based only on degree centrality utility achieves only $78 \%$ of delivery ratio.

\subsection{Lapland dataset}

We now discuss the routing performance of ML-SOR compared to the other routing schemes, starting from Lapland dataset. Delivery ratio, overhead cost and average latency for this trace are shown in Fig. 4. By analyzing delivery ratio, we observe that all algorithms deliver more packets to the destinations when the TTL increases. However, as the TTL becomes high the increment in the delivery ratio is marginal, since the capacity of the network to forward packets becomes the performance bottleneck. Epidemic routing outperforms all the other protocols with the highest delivery ratio, achieving $61 \%$ of message delivery. We can observe that its overhead cost having a value of 15 on average is also very high because of the large amount of message replicas injected into the network. That is why an opportunistic protocol with a high delivery capability, as in the case of Epidemic routing, but with a lower cost would be the right choice in order to save energy. As can be seen, PRoPHET is a good candidate, since it reduces overhead cost, with a delivery ratio slightly lower than Epidemic routing. Moreover, PRoPHET outperforms all social-based protocols in terms of message delivery. Adding probabilities to the decision making, as in the case of PRoPHET, works better than social information is this mobility scenario.

In terms of overhead cost, however, PRoPHET costs much more than social-based protocols. On the contrary, multilayer social information included by ML-SOR in the forwarding decision reduces notably overhead. ML-SOR shows the lowest overhead cost while maintaining a delivery ratio which is about $10 \%$ less than PRoPHET's delivery ratio. As far as Bubble Rap and H-Bubble Rap are concerned, the hybrid version of Bubble Rap outperforms classic Bubble Rap both in terms of delivery ratio and overhead cost, showing a delivery ratio comparable to ML-SOR. These results demon-

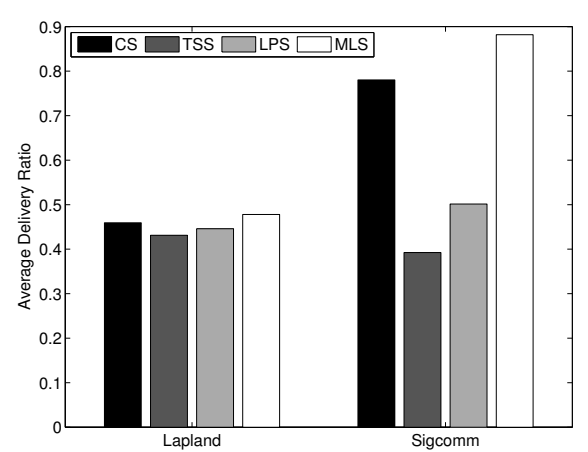

Figure 3: Routing performance of utility scores.

strate that protocols exploiting multi-layer social network information are able to select more efficient paths.

By looking at average latency, for low TTLs (1 hour and 2 hours) all protocols show a similar average latency. As TTL is increased, Epidemic routing and PRoPHET are able to deliver messages faster than the other protocols. The reason is that they replicate more packets than the other algorithms, as can be seen from their overhead costs, thus reducing delivery delay. H-Bubble Rap, on the contrary, even if it transmits more messages than ML-SOR, has an higher average latency for each TTL value, while Bubble Rap performs slightly better.

\subsection{Sigcomm dataset}

In Fig. 5, we present the results of the evaluation of the same routing strategies on Sigcomm data. When compared to Lapland dataset, the Sigcomm overall delivery ratio is higher, with values that achieve more than $95 \%$ of message delivery. Here the number of nodes is higher, hence the possibilities of forwarding and delivery are higher as well. Among all algorithms, Epidemic routing again has the highest delivery ratio. However, for most TTLs, ML-SOR and H-Bubble Rap perform the same as Epidemic routing. With a higher number of nodes, the probabilistic strategy of PRoPHET performs worse, even if for high TTLs (10, 11 and 12 hours) it achieves Epidemic routing performance. Once again, as for Lapland dataset, Bubble Rap shows the lowest delivery ratio, achieving around $83 \%$ of message delivery. This means that ML-SOR social metric is able to improve the performance of Bubble Rap, both in the case of ML-SOR which does not consider communities to drive routing decisions and of $\mathrm{H}$-Bubble Rap which is communitybased.

In terms of cost, we can observe that among the algorithms considered Epidemic routing has the highest cost. Again, this is because Epidemic routing generates many message replicas. In contrast, the other four algorithms are more greedy in replication. For low TTLs (1, 2, and 3 hours), PRoPHET has a lower cost than Bubble Rap. When TTL increases, PRoPHET costs much more than the three social-based schemes. Moreover, ML-SOR and H-Bubble Rap, confirm that the use of the multi-layer social metric not only gives better performance than Bubble Rap that uses only contact network social information, but is also able to find more effective relays than PRoPHET. We can further note that H-Bubble Rap has the lowest overhead cost. 

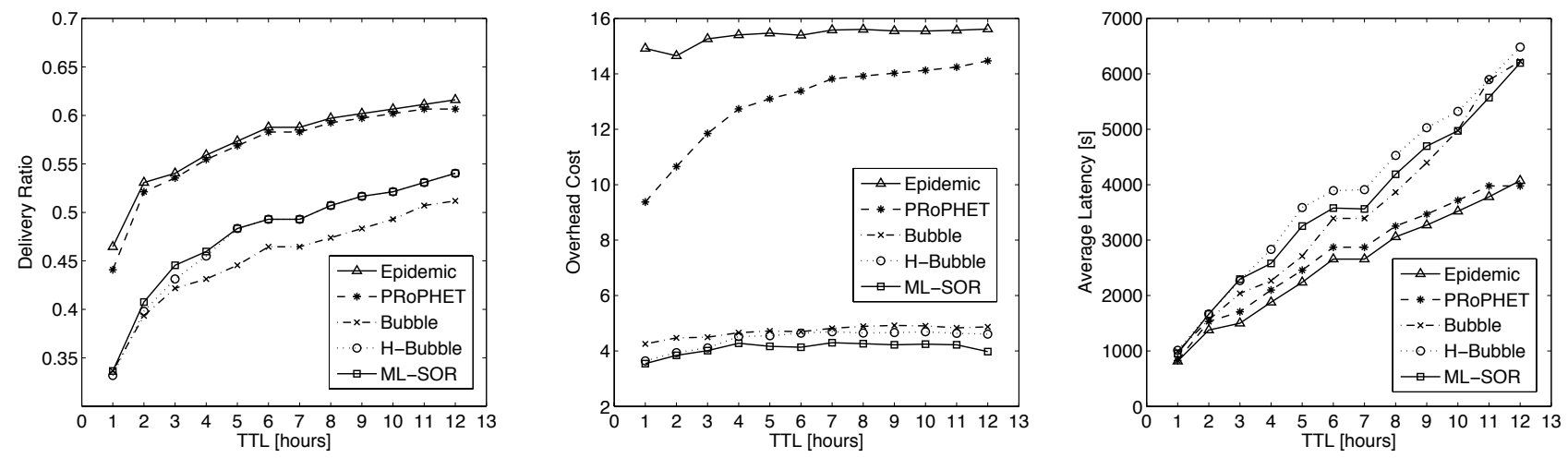

Figure 4: Comparison of ML-SOR with other algorithms in terms of delivery ratio, overhead and latency (Lapland dataset).
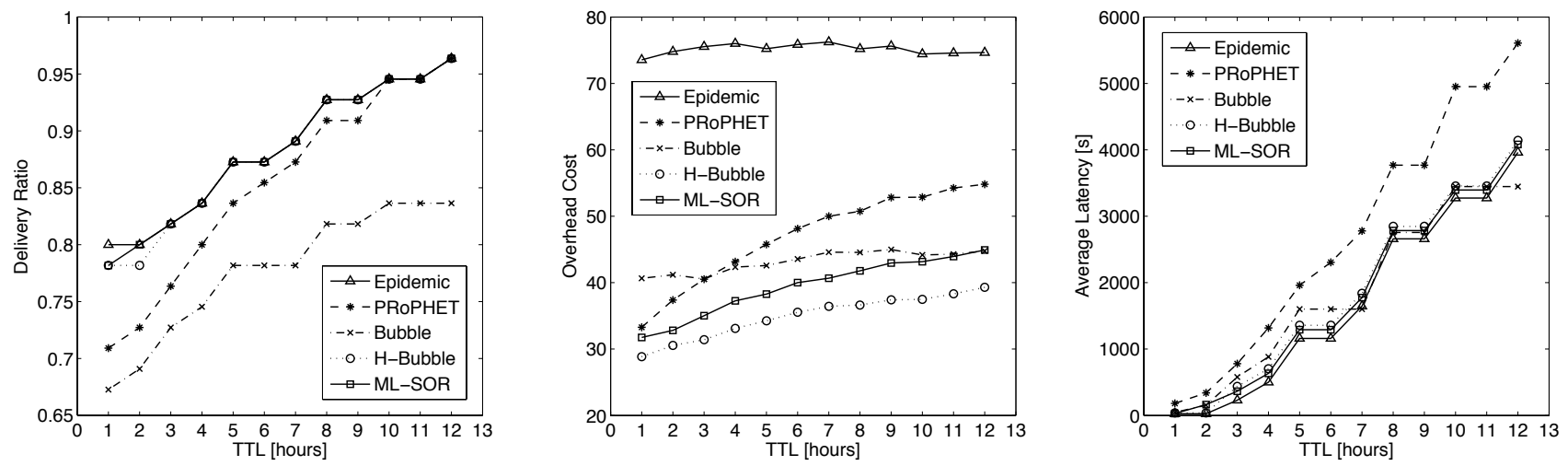

Figure 5: Comparison of ML-SOR with other algorithms in terms of delivery ratio, overhead and latency (Sigcomm dataset).

In Sigcomm scenario, the multi-layer social network metric combined with community detection is more effective than ML-SOR.

As we can see from average latency, among all protocols Epidemic routing performs the best except for TTL set to 12 hours where Bubble Rap performs better, while PRoPHET is characterized by the worst performance. PRoPHET behavior is completely different from Lapland dataset, where its average latency was similar to Epidemic routing. Here, also social-based schemes outperform PRoPHET. As for delivery ratio, Epidemic routing, H-Bubble Rap and ML-SOR performance is very similar. In particular, the multi-layer social metric of ML-SOR and H-Bubble Rap works quite well producing a latency lower than classic Bubble Rap for most TTLs.

\section{DISCUSSION}

ML-SOR is a social-based algorithm exploiting multiple social networks to perform routing in a mobile opportunistic network. This algorithm is deployed in a distributed manner and exchanges only small amounts of information. Considering that energy consumption is a fundamental issue in opportunistic networks, the reduction of unnecessary message-forwarding will conserve energy and hence improve network performance. ML-SOR takes advantage of multiple social contexts in order to reduce the number of transmissions.

Our results, although limited to two datasets, describe the behavior of our protocol in two environments where node mobility and the amount of social information is different. Considering OSN information, for example, each Lapland mobile node has a Facebook profile while in Sigcomm, a small subset of mobile nodes does not does not has Facebook information (probably for privacy issues or simply because they do not use Facebook). On the contrary, considering interests, both in Lapland and in Sigcomm each node has at least one interest. Our plans for future work include the analysis of other datasets with different connectivity patterns and social network layers such as Twitter or LinkedIn, in order to gain further validation of our forwarding scheme.

\section{CONCLUSIONS}

In this paper, we presented a model for the representation of multi-layer social networks together with a novel opportunistic routing protocol, ML-SOR, that uses this model to select nodes to act as message relays. The results of the performance evaluation of ML-SOR showed that more stable social information provided by several social network layers is able to augment available partial contact information improving message forwarding. In particular, we compared our protocol to Epidemic routing, PRoPHET, Bubble Rap and 
a hybrid version of Bubble Rap, called H-Bubble Rap, which uses the same metric of ML-SOR and is community-based. Testing the Lapland trace and Sigcomm trace, we showed that by combining multiple social information messages can be delivered with high probability while keeping overhead ratio very small. Additionally, in Sigcomm dataset, delivery performance may be achieved equal to Epidemic routing but with significantly reduced overhead cost.

\section{REFERENCES}

[1] S. Ahmed and S. S. Kanhere. Hubcode: message forwarding using hub-based network coding in delay tolerant networks. In Proceedings of the 12th ACM international conference on Modeling, analysis and simulation of wireless and mobile systems, MSWiM '09, pages 288-296, New York, NY, USA, 2009. ACM.

[2] G. Bigwood and T. Henderson. Bootstrapping opportunistic networks using social roles. In World of Wireless, Mobile and Multimedia Networks (WoWMoM), 2011 IEEE International Symposium on $a$, pages $1-6$, june 2011.

[3] V. Cerf, S. Burleigh, A. Hooke, L. Torgerson, R. Durst, R. Scott, and K. Fall. Delay-tolerant networking architecture. http://tools.ietf.org/html/rfc4838, April 2007.

[4] R. I. Ciobanu, C. Dobre, and V. Cristea. SPRINT: social prediction-based opportunistic routing. In Proceedings of 7th IEEE WoWMoM workshop on autonomic and opportunistic communications, AOC 2013, pages 1161-1166, Madrid, Spain, May 2013.

[5] E. Daly and M. Haahr. Social network analysis for information flow in disconnected delay-tolerant manets. Mobile Computing, IEEE Transactions on, 8(5):606 -621, may 2009.

[6] F. De Rango and F. Monteverdi. Social and dynamic graph-based scalable routing protocol in a DTN network. In Performance Evaluation of Computer and Telecommunication Systems (SPECTS), 2012 International Symposium on, pages 1-8. IEEE, 2012.

[7] F. De Rango, A. Socievole, A. Scaglione, and S. Marano. Novel activity-based metrics for efficient forwarding over online and detected social networks. In Wireless Communications and Mobile Computing Conference (IWCMC), 2013 9th International, July 2013.

[8] K. Fall. A delay-tolerant network architecture for challenged internets. In Proceedings of the 2003 conference on Applications, technologies, architectures, and protocols for computer communications, SIGCOMM '03, pages 27-34, New York, NY, USA, 2003. ACM.

[9] S. Gaito, G. P. Rossi, and M. Zignani. Facencounter: bridging the gap between offline and online social networks. In Proceedings of 8th international conference on signal image technology and Internet based systems (SITIS), pages 768-775, november 2012.

[10] P. Hui, A. Chaintreau, J. Scott, R. Gass, J. Crowcroft, and C. Diot. Pocket switched networks and human mobility in conference environments. In Proceedings of the 2005 ACM SIGCOMM workshop on Delay-tolerant networking, WDTN '05, pages 244-251, New York, NY, USA, 2005. ACM.
[11] P. Hui, J. Crowcroft, and E. Yoneki. Bubble rap: Social-based forwarding in delay-tolerant networks. Mobile Computing, IEEE Transactions on, 10(11):1576-1589, 2011.

[12] A. Keränen, J. Ott, and T. Kärkkäinen. The ONE simulator for DTN protocol evaluation. In Proceedings of the 2nd International Conference on Simulation Tools and Techniques, Simutools '09, pages 55:1-55:10, ICST, Brussels, Belgium, Belgium, 2009.

[13] A. Lindgren, A. Doria, and O. Schelén. Probabilistic routing in intermittently connected networks, 2004.

[14] A. Mashhadi, S. Ben Mokhtar, and L. Capra. Habit: Leveraging human mobility and social network for efficient content dissemination in delay tolerant networks. In World of Wireless, Mobile and Multimedia Networks Workshops, 2009. WoWMoM 2009. IEEE International Symposium on a, pages 1 -6 , june 2009.

[15] A. Mtibaa, A. Chaintreau, J. LeBrun, E. Oliver, A.-K. Pietilainen, and C. Diot. Are you moved by your social network application? In Proceedings of the first workshop on Online social networks, WOSN '08, pages 67-72, New York, NY, USA, 2008. ACM.

[16] A. Mtibaa, M. May, C. Diot, and M. Ammar. Peoplerank: Social opportunistic forwarding. In INFOCOM, 2010 Proceedings IEEE, pages $1-5$, march 2010.

[17] L. Pelusi, A. Passarella, and M. Conti. Opportunistic networking: data forwarding in disconnected mobile ad hoc networks. Communications Magazine, IEEE, 44(11):134 -141, november 2006.

[18] A.-K. Pietilainen and C. Diot. CRAWDAD data set thlab/sigcomm2009 (v. 2012-07-15). Downloaded from http://crawdad.cs.dartmouth.edu/thlab/sigcomm2009, July 2012.

[19] A.-K. Pietiläinen, E. Oliver, J. LeBrun, G. Varghese, and C. Diot. Mobiclique: middleware for mobile social networking. In Proceedings of the 2nd ACM workshop on Online social networks, WOSN '09, pages 49-54, New York, NY, USA, 2009. ACM.

[20] A. Socievole, F. De Rango, and S. Marano. Face-to-face with Facebook friends: using online friendlists for routing in opportunistic networks. accepted at the 24th IEEE International Symposium on Personal, Indoor and Mobile Radio Communications (PIMRC 2013).

[21] A. Socievole and S. Marano. Exploring user sociocentric and egocentric behaviors in online and detected social networks. In Future Internet Communications (BCFIC), 2012 2nd Baltic Congress on, pages $140-147$, april 2012.

[22] A. Vahdat and D. Becker. Epidemic routing for Partially-Connected ad hoc networks. Technical report, Duke University, April 2000.

[23] K. Xu, V. Li, and J. Chung. Exploring centrality for message forwarding in opportunistic networks. In Wireless Communications and Networking Conference (WCNC), 2010 IEEE, pages 1 -6, april 2010.

[24] E. Yoneki and F. B. Abdesslem. Finding a data blackhole in bluetooth scanning. ExtremeCom, 2009. 